\title{
ON THE LELONG-DEMAILLY NUMBERS OF PLURISUBHARMONIC CURRENTS
}

\author{
NOUREDDINE GHILOUFI
}

\begin{abstract}
In this note we study the existence of the Lelong-Demailly number of a negative plurisubharmonic current with respect to a positive plurisubharmonic function on an open subset of $\mathbb{C}^{n}$. Then we establish some estimates of the Lelong-Demailly numbers of positive or negative plurisubharmonic currents.
\end{abstract}

Sur les Nombres de Lelong-Demailly des courants plurisousharmoniques.

RÉSumÉ. Dans cette note, on étudie l'existence du nombre de LelongDemailly d'un courant négatif plurisousharmonique relativement à une fonction positive plurisousharmonique sur un ouvert de $\mathbb{C}^{n}$ puis on donne quelques estimations des nombres de Lelong-Demailly des courants positifs ou négatifs plurisousharmoniques.

\section{VERSION FRANÇAISE ABRÉGÉE}

L'existence des nombres de Lelong des courants positifs a été résolu par P. Lelong dans les années 1950 pour le cas des courants fermés, puis ce résultat a été étendu par Skoda au cas des courants positifs plurisousharmoniques. En revanche, il existe des courants négatifs plurisousharmoniques qui n'admettent pas de nombres de Lelong, on peut voir par exemple que $\log \left(\left|z_{2}\right|^{2}\right)\left[z_{1}=0\right]$ est un exemple de courant négatif plurisousharmonique de bidimension $(1,1)$ sur la boule unité de $\mathbb{C}^{2}$ qui n'admet pas de nombre de Lelong en 0.

Le principal objectif de cette note est de traiter le problème de l'existence des nombres de Lelong généralisés introduits par Demailly [1], d'un courant négatif plurisousharmonique $T$ de bidimension $(p, p)$ sur un ouvert $\Omega$ de $\mathbb{C}^{n}$; pour cela on note $\operatorname{PSH}(T, \Omega)$ l'ensemble des fonctions $\varphi$ positives plurisousharmoniques semi-exhaustives dont le $\log a r i t h m e \log \varphi$ est plurisousharmonique sur $\Omega$, et telles que le produit extérieur $T \wedge\left(d d^{c} \varphi\right)^{p}$ soit bien défini. Le nombre de Lelong-Demailly de $T$ relativement à un poids $\varphi$ tel que $\varphi \in$ $\operatorname{PSH}(T, \Omega)$ est $\nu(T, \varphi):=\lim _{r \rightarrow 0^{+}} \nu(T, \varphi, r)$, où $\nu(T, \varphi,$.$) est la fonction$

2000 Mathematics Subject Classification. 32U25; 32U40; 32U05.

Key words and phrases. Lelong number, plurisubharmonic current, plurisubharmonic function. 
définie par

$$
\nu(T, \varphi, r):=\frac{1}{r^{p}} \int_{\{\varphi<r\}} T \wedge\left(d d^{c} \varphi\right)^{p} .
$$

Le résultat principal de cette note est:

Théorème 1. Soient $T$ un courant négatif plurisousharmonique de bidimension $(p, p)$ sur $\Omega$ et $\varphi \in \operatorname{PSH}(T, \Omega)$. Si la fonction $t \longmapsto \frac{\nu\left(d d^{c} T, \varphi, t\right)}{t}$ est intégrable au voisinage de 0 , alors le nombre de Lelong-Demailly $\nu(T, \varphi) d u$ courant $T$ relativement $\grave{a} \varphi$ existe.

\section{INTRODUCTION}

The existence of Lelong numbers of positive currents was proved by P. Lelong in the 1950's in the case of closed currents, then this result was extended by H. Skoda to the case of positive plurisubharmonic currents. However, there are negative plurisubharmonic currents which do not admit Lelong numbers, for example $-\left(-\log \left(\left|z_{2}\right|^{2}\right)\right)^{\epsilon}\left[z_{1}=0\right]$ is a negative plurisubharmonic current of bidimension $(1,1)$ on the unit ball of $\mathbb{C}^{2}$, which admits no Lelong number at 0 for all $0<\epsilon \leq 1$.

The main purpose of the first part of this note is to study the existence of generalized Lelong numbers, introduced by Demailly [1], in the case of negative plurisubharmonic currents of bidimension $(p, p)$ on an open set $\Omega$ of $\mathbb{C}^{n}$. In the second part, we give some proprieties of the Lelong-Demailly numbers of positive or negative plurisubharmonic currents. In particular, we prove that the Lelong-Demailly numbers do not depend to the system of coordinates. To this aim, we consider a non-negative plurisubharmonic function $\varphi$ on $\Omega$ such that $\log \varphi$ is plurisubharmonic on $\{\varphi>0\}$ and for every $r>0, r_{2}>r_{1}>0$, we set

$$
\begin{aligned}
& B_{\varphi}(r):=\{z \in \Omega ; \varphi(z)<r\}, \\
& B_{\varphi}\left(r_{1}, r_{2}\right):=\left\{z \in \Omega ; r_{1} \leq \varphi(z)<r_{2}\right\}=B_{\varphi}\left(r_{2}\right) \backslash B_{\varphi}\left(r_{1}\right) \\
& \beta_{\varphi}:=d d^{c} \varphi=\frac{i}{\pi} \partial \bar{\partial} \varphi, \quad \alpha_{\varphi}:=d d^{c} \log \varphi \text { on }\{\varphi>0\} .
\end{aligned}
$$

We assume that $\varphi$ is semi-exhaustive, i.e. there exists $R=R(\varphi)>0$ such that $B_{\varphi}(R)$ is relatively compact in $\Omega$. If $S$ is a positive (or negative) current of bidimension $(p, p)$ on the set $\Omega$ then we denote $\operatorname{PSH}(S, \Omega)$ the set of nonnegative semi-exhaustive plurisubharmonic functions $\varphi$ such that $\log \varphi$ is also plurisubharmonic on $\Omega$ and the exterior product $S \wedge\left(d d^{c} \varphi\right)^{p}$ is well defined. Finally, we denote by $\mathcal{I}_{S}(\varphi):=\left\{k>0 ; \varphi^{k} \in \operatorname{PSH}(S, \Omega)\right\}$ for every $\varphi \in \operatorname{PSH}(S, \Omega)$; in particular, if $\varphi$ is $\mathcal{C}^{2}$ then $\mathcal{I}_{S}(\varphi) \supset[1,+\infty[$ for every current $S$.

The Lelong-Demailly number of $S$ relatively to the weight $\varphi$ is $\nu(S, \varphi):=$ $\lim _{r \rightarrow 0^{+}} \nu(S, \varphi, r)$ where $\nu(S, \varphi,$.$\left.) is the function defined on \right] 0, R(\varphi)[$ by

$$
\nu(S, \varphi, r):=\frac{1}{r^{p}} \int_{B_{\varphi}(r)} S \wedge \beta_{\varphi}^{p} .
$$


The classical Lelong number is given by the choice $\varphi(z)=\varphi_{0}(z):=|z|^{2}$.

Example 1. Let $S_{\epsilon}\left(z_{1}, z_{2}\right)=\left(\left|z_{2}\right|^{2 \epsilon}-1\right)\left[z_{1}=0\right]$ where $\epsilon>0$. Then $S_{\epsilon}$ is a negative plurisubharmonic current of bidimension $(1,1)$ on the unit ball $\mathbb{B}$ of $\mathbb{C}^{2}, \varphi_{0} \in \operatorname{PSH}\left(S_{\epsilon}, \mathbb{B}\right)$ and $\left.\mathcal{I}_{S_{\epsilon}}\left(\varphi_{0}\right)=\mathcal{I}_{d d^{c} S_{\epsilon}}\left(\varphi_{0}\right)=\right] 0,+\infty[$.

Proof. A simple computation proves that

$$
S_{\epsilon} \wedge d d^{c}\left(\varphi_{0}^{k}\right)=\frac{k^{2}}{\pi}\left(\left|z_{2}\right|^{2 \epsilon}-1\right)\left|z_{2}\right|^{2(k-1)} i d z_{2} \wedge d \bar{z}_{2} \wedge\left[z_{1}=0\right]
$$

and $d d^{c} S_{\epsilon}=\frac{\epsilon^{2}}{\pi}\left|z_{2}\right|^{2(\epsilon-1)} i d z_{2} \wedge d \bar{z}_{2} \wedge\left[z_{1}=0\right]$, hence $S_{\epsilon} \wedge d d^{c}\left(\varphi_{0}^{k}\right)$ is well defined for all $k>0$. Therefore, $\left.\mathcal{I}_{S_{\epsilon}}\left(\varphi_{0}\right)=\mathcal{I}_{d d^{c} S_{\epsilon}}\left(\varphi_{0}\right)=\right] 0,+\infty[$. One can check easily that

$$
\nu\left(S_{\epsilon}, \varphi_{0}^{k}, r\right)=2 k^{2}\left(\frac{r^{\epsilon / k}}{\epsilon+k}-\frac{1}{k}\right) \quad \text { and } \quad \nu\left(d d^{c} S_{\epsilon}, \varphi_{0}, r\right)=2 \epsilon r^{\epsilon},
$$

hence $\nu\left(S_{\epsilon}, \varphi_{0}^{k}\right)=-2 k$.

\section{MAin RESUlt}

In the following, we will use a Lelong-Jensen formula proved by Demailly [1. In [2, Toujani used an analogue of this formula to prove the existence of the directional Lelong-Demailly numbers of positive plurisubharmonic currents.

Lemma 1. (See 1 or 2]) Let $S$ be a positive or negative plurisubharmonic current of bidimension $(p, p)$ on $\Omega$ and $\varphi \in \operatorname{PSH}(S, \Omega)$. Then, for all $0<$ $r_{1}<r_{2}<R(\varphi)$,

$$
\begin{aligned}
\nu\left(S, \varphi, r_{2}\right)-\nu\left(S, \varphi, r_{1}\right)= & \frac{1}{r_{2}^{p}} \int_{B_{\varphi}\left(r_{2}\right)} S \wedge \beta_{\varphi}^{p}-\frac{1}{r_{1}^{p}} \int_{B_{\varphi}\left(r_{1}\right)} S \wedge \beta_{\varphi}^{p} \\
= & \int_{B_{\varphi}\left(r_{1}, r_{2}\right)} S \wedge \alpha_{\varphi}^{p} \\
& +\int_{r_{1}}^{r_{2}}\left(\frac{1}{t^{p}}-\frac{1}{r_{2}^{p}}\right) d t \int_{B_{\varphi}(t)} d d^{c} S \wedge \beta_{\varphi}^{p-1} \\
& +\left(\frac{1}{r_{1}^{p}}-\frac{1}{r_{2}^{p}}\right) \int_{0}^{r_{1}} d t \int_{B_{\varphi}(t)} d d^{c} S \wedge \beta_{\varphi}^{p-1} .
\end{aligned}
$$

According to Lemma 1, if $S$ is positive plurisubharmonic then $\nu(S, \varphi,$.$) is$ a non-negative increasing function on $] 0, R(\varphi)\left[\right.$, so $\nu(S, \varphi):=\lim _{r \rightarrow 0^{+}} \nu(S, \varphi, r)$ exists.

It is well known that if $S$ is a positive plurisubharmonic current, then for every $\varphi \in \operatorname{PSH}(S, \Omega)$ the function $t \longmapsto \frac{\nu\left(d d^{c} S, \varphi, t\right)}{t}$ is integrable in the neighborhood of 0 . Throughout this note, for every negative plurisubharmonic current $T$ on $\Omega$, we say that a function $\varphi \in \operatorname{PSH}(T, \Omega)$ satisfies condition $(C)$ if the function $t \longmapsto \frac{\nu\left(d d^{c} T, \varphi, t\right)}{t}$ is integrable on a neighborhood of 0 . In particular, if $\varphi$ satisfies condition $(C)$, we must have $\nu\left(d d^{c} T, \varphi\right)=0$. 
Now we state the main result concerning the case of negative plurisubharmonic currents.

Theorem 1. Let $T$ be a negative plurisubharmonic current of bidimension $(p, p)$ on $\Omega$ and $\varphi \in \operatorname{PSH}(T, \Omega)$ satisfying condition $(C)$. Then, the LelongDemailly number $\nu(T, \varphi)$ of the current $T$ relatively to $\varphi$ exists.

Proof. For every $0<r<R(\varphi)$, we set

$f(r)=\frac{1}{r^{p}} \int_{B_{\varphi}(r)} T \wedge \beta_{\varphi}^{p}+\frac{1}{r^{p}} \int_{0}^{r} d t \int_{B_{\varphi}(t)} d d^{c} T \wedge \beta_{\varphi}^{p-1}-\int_{0}^{r} \frac{d t}{t^{p}} \int_{B_{\varphi}(t)} d d^{c} T \wedge \beta_{\varphi}^{p-1}$.

Thanks to condition $(C)$, the function $f$ is well defined and non-positive on ] $0, R(\varphi)[$. Indeed,

$$
f(r)=\nu(T, \varphi, r)+\int_{0}^{r}\left(\frac{t^{p}}{r^{p}}-1\right) \frac{\nu\left(d d^{c} T, \varphi, t\right)}{t} d t \leq 0
$$

because the function $\nu\left(d d^{c} T, \varphi,.\right)$ is non-negative on $] 0, R(\varphi)[$.

For $0<r_{1}<r_{2}<R(\varphi)$ we set $A\left(r_{1}, r_{2}\right):=f\left(r_{2}\right)-f\left(r_{1}\right)$. The current $T$ is negative plurisubharmonic, thus lemma 1 gives

$$
\begin{aligned}
A\left(r_{1}, r_{2}\right)= & \nu\left(T, \varphi, r_{2}\right)-\nu\left(T, \varphi, r_{1}\right)+\frac{1}{r_{2}^{p}} \int_{0}^{r_{2}} t^{p-1} \nu\left(d d^{c} T, \varphi, t\right) d t \\
& -\frac{1}{r_{1}^{p}} \int_{0}^{r_{1}} t^{p-1} \nu\left(d d^{c} T, \varphi, t\right) d t-\int_{r_{1}}^{r_{2}} \frac{\nu\left(d d^{c} T, \varphi, t\right)}{t} d t \\
= & \int_{B_{\varphi}\left(r_{1}, r_{2}\right)} T \wedge \alpha_{\varphi}^{p} \leq 0 .
\end{aligned}
$$

Therefore, $f$ is a non-positive decreasing function on $] 0, R(\varphi)[$, and this implies the existence of the limit $\varrho:=\lim _{r \rightarrow 0^{+}} f(r)$. The hypothesis of integrability of $\nu\left(d d^{c} T, \varphi, t\right) / t$ and the fact that $\left(t^{p} / r^{p}-1\right)$ is uniformly bounded give

$$
\lim _{r \rightarrow 0^{+}} \int_{0}^{r}\left(\frac{t^{p}}{r^{p}}-1\right) \frac{\nu\left(d d^{c} T, \varphi, t\right)}{t} d t=0 .
$$

Therefore, $\varrho=\lim _{r \rightarrow 0^{+}} f(r)=\lim _{r \rightarrow 0^{+}} \nu(T, \varphi, r)=\nu(T, \varphi)$.

Remark 1. If $T$ is a positive (resp. negative) plurisubharmonic current of bidimension $(p, p)$ on $\Omega$ and $\varphi \in \operatorname{PSH}(T, \Omega)$ (resp. satisfying condition $(C)$ ) then the extension $\widetilde{T \wedge \alpha_{\varphi}^{p}}$ of $T \wedge \alpha_{\varphi}^{p}$ by 0 over the compact set $\{\varphi=0\}$ exists and we have

$$
\int_{B_{\varphi}(r)} \widetilde{T \wedge \alpha_{\varphi}^{p}}=f(r)-\nu(T, \varphi) .
$$

Indeed, it suffice to use the lemma 1 to prove that $\int_{B_{\varphi}(\epsilon, r)} T \wedge \alpha_{\varphi}^{p}$ is uniformly bounded and then tend $\epsilon$ to 0 to prove the equality of the remark. A natural question arises: does the existence of the Lelong-Demailly number of the negative plurisubharmonic current $T$ implies the existence of $\widetilde{T \wedge \alpha_{\varphi}^{p}}$ ? so condition $(C)$ will be necessary in Theorem 1 . 
In the following proposition, we give some properties of Lelong-Demailly numbers in both cases of negative or positive plurisubharmonic currents.

Proposition 1. Let $T$ be a positive or negative plurisubharmonic current of bidimension $(p, p)$ on $\Omega$ and $\varphi \in \operatorname{PSH}(T, \Omega)$. Then, for every $k \in \mathcal{I}_{T}(\varphi)$ and every $r \in] 0, R(\varphi)[$, we have

$$
\nu\left(T, \varphi^{k}, r^{k}\right)=k^{p}\left[\nu(T, \varphi, r)+\int_{0}^{r} \frac{\nu\left(d d^{c} T, \varphi, t\right)}{t}\left(\frac{t^{p}}{r^{p}}-\frac{t^{k p}}{r^{k p}}\right) d t\right] .
$$

In particular, if $T$ is negative, then $\nu(T, \varphi)$ exists if and only if for all $k \in$ $\mathcal{I}_{T}(\varphi), \nu\left(T, \varphi^{k}\right)$ exists.

Furthermore, in both cases (with the assumption that $\nu(T, \varphi)$ exists if $T$ is negative) we have

$$
\nu\left(T, \varphi^{k}\right)=k^{p} \nu(T, \varphi) .
$$

The previous equality is due to Demailly in the case of closed positive currents.

Proof. Let $\epsilon>0$ be sufficiently small. By replacing $\varphi$ with $\varphi_{\epsilon}=\varphi+\epsilon$ and $\psi:=\varphi^{k}$ with $\psi_{\epsilon}=\varphi_{\epsilon}^{k}$, when $T$ is negative (respectively positive), Equality (2.2) (resp. Equality (2.1)) gives for $0<r_{1}<\epsilon<r<R(\varphi)$

$$
\begin{array}{r}
\nu\left(T, \varphi_{\epsilon}, r\right)=\int_{B_{\varphi_{\epsilon}}(\epsilon, r)} T \wedge \alpha_{\varphi_{\epsilon}}^{p}-\frac{1}{r^{p}} \int_{\epsilon}^{r} t^{p-1} \nu\left(d d^{c} T, \varphi_{\epsilon}, t\right) d t \\
+\int_{\epsilon}^{r} \frac{\nu\left(d d^{c} T, \varphi_{\epsilon}, t\right)}{t} d t
\end{array}
$$

and

$$
\begin{aligned}
\nu\left(T, \psi_{\epsilon}, r^{k}\right) & =\int_{B_{\psi_{\epsilon}}\left(\epsilon^{k}, r^{k}\right)} T \wedge \alpha_{\psi_{\epsilon}}^{p}-\frac{1}{r^{k p}} \int_{\epsilon^{k}}^{r^{k}} t^{p-1} \nu\left(d d^{c} T, \psi_{\epsilon}, t\right) d t \\
& =\int_{B_{\psi_{\epsilon}}\left(\epsilon^{k}, r^{k}\right)} T \wedge \int_{\epsilon^{k}}^{r^{k}} \frac{\nu\left(d d^{c} T, \psi_{\epsilon}, t\right)}{t} d t \\
& =k^{p} \int_{B_{\varphi_{\epsilon}}(\epsilon, r)} T \wedge \alpha_{\varphi_{\epsilon}}^{p}-\frac{k^{p}}{r^{k p}} \int_{\epsilon}^{r} s^{k p-1} \nu\left(d d^{c} T, \psi_{\epsilon}, s^{k}\right) d s \\
& +k \int_{\epsilon}^{r} \frac{\nu\left(d d^{c} T, \psi_{\epsilon}, s^{k}\right)}{s} d s \\
& =k^{p}\left(\nu\left(T, \varphi_{\epsilon}, r\right)+\frac{1}{r^{p}} \int_{\epsilon}^{r} \frac{t^{p-1} \nu\left(d d^{c}, s\right) d s}{t^{c}} \frac{\nu\left(d d^{c} T, \varphi_{\epsilon}, s\right)}{s} d s\right) \\
& =k^{p}\left(\nu\left(T, \varphi_{\epsilon}, r\right)+\int_{\epsilon}^{r} \frac{\nu\left(d d^{c} T, \varphi_{\epsilon}, t\right)}{t}\left(\frac{t^{p}}{r^{k p}}-\frac{t^{k p}}{r^{k p}}\right) d t\right) .
\end{aligned}
$$


Here we have used successively Equality (2.4) with $\psi_{\epsilon}$ instead of $\varphi_{\epsilon}$ in the first equality, then the change of variable $t=s^{k}$, next the fact that $\nu\left(d d^{c} T, \psi_{\epsilon}, s^{k}\right)=k^{p-1} \nu\left(d d^{c} T, \varphi_{\epsilon}, s\right)$ (equality proved by Demailly in the case of closed positive currents), and finally Equality (2.4).

When $\epsilon \rightarrow 0$, Equality (2.5) implies Equality (2.3).

Thanks to Equality (2.3), for every $r \in] 0, R(\varphi)[$, we have

$$
\nu(T, \varphi, r)-\frac{1}{k^{p}} \nu\left(T, \varphi^{k}, r^{k}\right)=-\int_{0}^{r} \nu\left(d d^{c} T, \varphi, t\right)\left(\frac{t^{p-1}}{r^{p}}-\frac{t^{k p-1}}{r^{k p}}\right) d t .
$$

The current $d d^{c} T$ is positive and closed, hence $\nu\left(d d^{c} T, \varphi,.\right)$ is a non-negative increasing function and $\nu\left(d d^{c} T, \varphi\right)=0$. We consider two disjoint cases:

- First case $k \geq 1$. We have

$$
\begin{aligned}
0 & \leq \int_{0}^{r} \nu\left(d d^{c} T, \varphi, t\right)\left(\frac{t^{p-1}}{r^{p}}-\frac{t^{k p-1}}{r^{k p}}\right) d t \\
& \leq \nu\left(d d^{c} T, \varphi, r\right) \int_{0}^{r}\left(\frac{t^{p-1}}{r^{p}}-\frac{t^{k p-1}}{r^{k p}}\right) d t \\
& =\nu\left(d d^{c} T, \varphi, r\right) \frac{k-1}{k p} .
\end{aligned}
$$

So

$$
-\frac{k-1}{k p} \nu\left(d d^{c} T, \varphi, r\right) \leq \nu(T, \varphi, r)-\frac{1}{k^{p}} \nu\left(T, \varphi^{k}, r^{k}\right) \leq 0
$$

- Second case $k<1$. A similar calculation shows that we have

$$
0 \leq \nu(T, \varphi, r)-\frac{1}{k^{p}} \nu\left(T, \varphi^{k}, r^{k}\right) \leq-\frac{k-1}{k p} \nu\left(d d^{c} T, \varphi, r\right) .
$$

In the two cases, both terms (of the right-hand and the left-hand) have the same limit 0 when $r \rightarrow 0^{+}$. This completes the proof of the proposition.

\section{Applications}

The following corollaries are immediate consequences of Theorem 1 and/or Proposition 1 ,

Corollary 1. Let $T$ be a negative plurisubharmonic current of bidimension $(p, p)$ on $\Omega$ and $\varphi \in \operatorname{PSH}(T, \Omega)$. If there exists $k \in \mathcal{I}_{T}(\varphi)$ such that the function $t \longmapsto \frac{\nu\left(d d^{c} T, \varphi, t^{\frac{1}{k}}\right)}{t}$ is integrable on a neighborhood of 0 , then the Lelong-Demailly number $\nu(T, \varphi)$ of $T$ relatively to $\varphi$ exists.

Proof. We can prove this corollary in two different ways:

- First way. Thanks to Proposition 1, to prove that $\nu(T, \varphi)$ exists, it suffices to show that $\nu\left(T, \varphi^{k}\right)$ exists; for this we observe that

$$
\frac{\nu\left(d d^{c} T, \varphi^{k}, t\right)}{t}=\frac{\nu\left(d d^{c} T, \varphi^{k},\left(t^{\frac{1}{k}}\right)^{k}\right)}{t}=k^{p-1} \frac{\nu\left(d d^{c} T, \varphi, t^{\frac{1}{k}}\right)}{t}
$$

is integrable on a neighborhood of 0 (hypothesis). Hence, thanks to Theorem 1, $\nu\left(T, \varphi^{k}\right)$ exists. 
- Second way. We remark that the integrability of $\frac{\nu\left(d d^{c} T, \varphi, t^{\frac{1}{k}}\right)}{t}$ is equivalent to the integrability of $\frac{\nu\left(d d^{c} T, \varphi, t\right)}{t}$. Hence, thanks to Theorem 1, $\nu(T, \varphi)$ exists. In fact if we take $t=s^{k}$ we obtain

$$
\int_{0}^{r_{0}} \frac{\nu\left(d d^{c} T, \varphi, t^{\frac{1}{k}}\right)}{t} d t=k \int_{0}^{r_{0}^{k}} \frac{\nu\left(d d^{c} T, \varphi, s\right)}{s} d s .
$$

Corollary 2. Let $T$ be a negative plurisubharmonic current of bidimension $(p, p)$ on $\Omega$ and $\varphi \in \operatorname{PSH}(T, \Omega)$ such that $\mathcal{I}_{T}(\varphi)$ is non bounded (this is always the case if $\varphi$ is $\mathcal{C}^{2}$ ). Then for every $\left.r \in\right] 0, R(\varphi)$ [ one has

$$
\nu(T, \varphi, r) \leq-\int_{0}^{r} \nu\left(d d^{c} T, \varphi, t\right) \frac{t^{p-1}}{r^{p}} d t .
$$

In particular,

$$
\nu(T, \varphi, r) \leq-\frac{1-s^{-p}}{p} \nu\left(d d^{c} T, s \varphi, r\right) \quad \forall s \geq 1 .
$$

Proof. Thanks to Proposition 1, for every $k \in \mathcal{I}_{T}(\varphi)$, we have

$$
u(k):=\frac{1}{k^{p}} \nu\left(T, \varphi^{k}, r^{k}\right)=\nu(T, \varphi, r)+\int_{0}^{r} \frac{\nu\left(d d^{c} T, \varphi, t\right)}{t}\left(\frac{t^{p}}{r^{p}}-\frac{t^{k p}}{r^{k p}}\right) d t .
$$

The function $u$ is non-positive and increasing on $\mathcal{I}_{T}(\varphi)$, so

$$
\lim _{k \rightarrow+\infty, k \in \mathcal{I}_{T}(\varphi)} u(k)=\nu(T, \varphi, r)+\int_{0}^{r} \nu\left(d d^{c} T, \varphi, t\right) \frac{t^{p-1}}{r^{p}} d t \leq 0 .
$$

If $s=1$ the inequality is clear. For $s>1$ we have

$$
\begin{aligned}
\nu(T, \varphi, r) \leq-\int_{0}^{r} \nu\left(d d^{c} T, \varphi, t\right) \frac{t^{p-1}}{r^{p}} d t & \leq-\int_{r / s}^{r} \nu\left(d d^{c} T, \varphi, t\right) \frac{t^{p-1}}{r^{p}} d t \\
& \leq-\frac{1-s^{-p}}{p} \nu\left(d d^{c} T, \varphi, \frac{r}{s}\right) .
\end{aligned}
$$

Theorem 2. Let $T$ be a positive or negative plurisubharmonic current of bidimension $(p, p)$ on $\Omega$ and $\varphi, \psi \in \operatorname{PSH}(T, \Omega)$ such that

$$
\liminf _{\varphi(z) \rightarrow 0} \frac{\log \psi(z)}{\log \varphi(z)} \geq \ell
$$

where $\mathcal{I}_{T}(\varphi)$ contains a neighborhood $\mathscr{V}(\ell)$ of $\ell$. One has

- $\nu(T, \psi) \geq \ell^{p} \nu(T, \varphi)$ if $T$ is positive.

- $\nu(T, \psi) \leq \ell^{p} \nu(T, \varphi)$ if $T$ is negative (with the assumption that $\psi$ satisfies condition $(C))$.

In particular, if $\log \psi(z) \sim \ell \log \varphi(z)$ for $\varphi(z) \in \mathscr{V}(0)$ then $\nu(T, \psi)=\ell^{p} \nu(T, \varphi)$. 
This type of theorem is called a "comparison theorem"; such a statement has been proved by Demailly in the case of positive closed currents. Furthermore, one can deduce the invariance of the Lelong-Demailly numbers under changes of coordinate systems.

Proof. One can replace $\psi$ by $\psi^{k}$ with $1<k \in \mathcal{I}_{T}(\psi)$, in order to assume that

$$
\liminf _{\varphi(z) \rightarrow 0} \frac{\log \psi(z)}{\log \varphi(z)}>\ell
$$

As a consequence

$$
\lim _{\varphi(z) \rightarrow 0} \frac{\psi(z)}{\varphi(z)^{\ell}}=0
$$

For $\epsilon>0$ small sufficiently, we set $\Psi_{\epsilon}:=\psi+\epsilon \varphi^{\ell} \underset{\varphi(z) \in \mathscr{V}(0)}{\sim} \epsilon \varphi^{\ell}$. Thanks to the dominated convergence theorem,

$$
\lim _{\epsilon \rightarrow 0} \frac{1}{r^{p}} \int_{\Psi_{\epsilon}<r} T \wedge \beta_{\Psi_{\epsilon}}^{p}=\frac{1}{r^{p}} \int_{\psi<r} T \wedge \beta_{\psi}^{p} .
$$

- First assume $T \geq 0$. As the function $\nu\left(T, \Psi_{\epsilon},.\right)$ is increasing then

$$
\begin{aligned}
\nu\left(T, \Psi_{\epsilon}, r\right) & =\frac{1}{r^{p}} \int_{\Psi_{\epsilon}<r} T \wedge \beta_{\Psi_{\epsilon}}^{p} \\
& \geq \lim _{\rho \rightarrow 0} \frac{1}{\rho^{p}} \int_{\Psi_{\epsilon}<\rho} T \wedge \beta_{\Psi_{\epsilon}}^{p}=\lim _{\rho \rightarrow 0} \frac{1}{\rho^{p}} \int_{\epsilon \varphi^{\ell}<\rho} T \wedge \beta_{\Psi_{\epsilon}}^{p} \\
& \geq \lim _{\rho \rightarrow 0} \frac{1}{\rho^{p}} \int_{\epsilon \varphi^{\ell}<\rho} T \wedge \beta_{\epsilon \varphi^{\ell}}^{p}=\nu\left(T, \varphi^{\ell}\right) .
\end{aligned}
$$

because $\Psi_{\epsilon} \sim \epsilon \varphi^{\ell}$ and $T \wedge\left(d d^{c}\left(\psi+\epsilon \varphi^{\ell}\right)\right)^{p} \geq T \wedge\left(\epsilon d d^{c}\left(\varphi^{\ell}\right)\right)^{p}$. If $\epsilon \rightarrow 0$, we obtain $\nu(T, \psi, r) \geq \nu\left(T, \varphi^{\ell}\right)$. Hence, if $r \rightarrow 0, \nu(T, \psi) \geq$ $\nu\left(T, \varphi^{\ell}\right)$.

- Now, assume $T \leq 0$. Thanks to Theorem 1, Equality (2.2) gives

$$
\begin{aligned}
\nu\left(T, \Psi_{\epsilon}, r\right)+\int_{0}^{r} \frac{\nu\left(d d^{c} T, \Psi_{\epsilon}, t\right)}{t}\left(\frac{t^{p}}{r^{p}}-1\right) d t & \leq \nu\left(T, \Psi_{\epsilon}\right) \\
& =\lim _{\rho \rightarrow 0} \frac{1}{\rho^{p}} \int_{\Psi_{\epsilon}<\rho} T \wedge \beta_{\Psi_{\epsilon}}^{p}
\end{aligned}
$$

and a similar calculation proves that

$$
\nu\left(T, \Psi_{\epsilon}, r\right)+\int_{0}^{r} \frac{\nu\left(d d^{c} T, \Psi_{\epsilon}, t\right)}{t}\left(\frac{t^{p}}{r^{p}}-1\right) d t \leq \nu\left(T, \varphi^{\ell}\right) .
$$

So if $\epsilon \rightarrow 0$ we obtain

$$
\nu(T, \psi, r)+\int_{0}^{r} \frac{\nu\left(d d^{c} T, \psi, t\right)}{t}\left(\frac{t^{p}}{r^{p}}-1\right) d t \leq \nu\left(T, \varphi^{\ell}\right) .
$$

When $r \rightarrow 0$, the last inequality gives $\nu(T, \psi) \leq \nu\left(T, \varphi^{\ell}\right)$. 
In the particular case $\log \psi(z) \sim \ell \log \varphi(z)$, for every $\epsilon>0$, there exists $\eta>0$ so that if $|\varphi(z)|<\eta$, then $\varphi(z)^{\ell(1+\epsilon)} \leq \psi(z) \leq \varphi(z)^{\ell(1-\epsilon)}$ and for $\epsilon$ small enough, $\ell(1+\epsilon), \ell(1-\epsilon) \in \mathscr{V}(\ell) \subset \mathcal{I}_{T}(\varphi)$. Therefore we can apply the previous inequalities to obtain $\ell^{p}(1+\epsilon)^{p} \nu(T, \varphi) \leq \nu(T, \psi) \leq \ell^{p}(1-\epsilon)^{p} \nu(T, \varphi)$ $\left(\right.$ resp. $\ell^{p}(1-\epsilon)^{p} \nu(T, \varphi) \leq \nu(T, \psi) \leq \ell^{p}(1+\epsilon)^{p} \nu(T, \varphi)$ in the case $T \geq 0$ (resp. $T \leq 0)$. Hence, when $\epsilon \rightarrow 0$, we obtain $\nu(T, \psi)=\ell^{p} \nu(T, \varphi)$.

\section{ACKNOWLEDGEMEnTS}

I thank Professor Jean-Pierre Demailly for several suggestions which enabled me to clarify and improve the original form of this note. I thank also Professor Khalifa Dabbek for useful discussions concerning this work.

\section{REFERENCES}

[1] J.-P. Demailly, Sur les nombres de Lelong associés à l'image directe d'un courant positif fermé, Annales de l'institut Fourier, tome 32, n² p37-66 (1982).

[2] M. Toujani, Nombre de Lelong directionnel d'un courant positif plurisousharmonique, C. R. Acad. Sci. Paris, Ser. I 343 p705-710 (2006).

E-mail address: noureddine.ghiloufi@fsg.rnu.tn

Department of Mathematics, Faculty of sciences of Gabès, University of Gabès, 6072 Gabès Tunisia. 